\title{
Elaboração de curva $A B C$ de medicamentos em uma unidade de saúde do município de Belém - PA
}

\author{
ABC curve elaboration of medicines in a Health Unit in Belém - PA \\ Elaboración de la curva $A B C$ de medicamentos en una unidad de salud en la ciudad de \\ Belém - PA \\ Jéssica Nayara Araújo da Costa ${ }^{1 *}$, Marcelo Fernandez Galvão Rodrigues ${ }^{2,}$ Priscila Gabriela de \\ Souza Braga ${ }^{3}$, Brianna Jandira Sousa dos Santos ${ }^{1}$, Luanny Paula Dias de Oliveira ${ }^{1}$, Ellen Carolina \\ de Vilhena Lima de Melo ${ }^{4}$, Valdenira Gonçalves da Silva ${ }^{5}$, Diandra Araújo da Luz ${ }^{6}$, Priscila de \\ Nazaré Quaresma Pinheiro6.
}

\section{RESUMO}

Objetivo: Elaborar a Curva $A B C$ de medicamentos em uma Unidade básica de saúde do município de BelémPA, demonstrando sua relevância para o controle de estoque eficiente. Métodos: Trata-se de um estudo descritivo transversal, que usou informações do gerenciamento de medicamentos utilizados na UBS, através de dados coletados das requisições mensais de medicamentos do período de setembro de 2017 a agosto de 2018. Os dados foram analisados no programa Microsoft Office Excelß 2013. Resultados: Dos 268 medicamentos padronizados, observou-se ausência de 105. Dos 112 do estoque da atenção básica, a classe A representou $18,42 \%$ destes medicamentos representando $69,76 \%$ dos custos, a classe $B 21,05 \%$ dos itens e representou $19,25 \%$ das despesas, a $C 60,53 \%$ dos itens com 10,99\% de custos. Na urgência e emergência, dos 51 medicamentos, as classes A e B corresponderam a $14,29 \%$ dos fármacos e a classe $C$ a $71,43 \%$. A demanda financeira, foi de: $68,93 \%$ (A), $20,26 \%$ (B) e a $C$ de $10,81 \%$. Conclusão: É importante investir no gerenciamento de medicamentos, para a racionalização dos recursos, da terapia farmacológica e evitar quebra do estoque.

Palavras-chave: Administração de materiais no hospital, Gestão em Saúde, Assistência Farmacêutica.

\begin{abstract}
Objective: To elaborate the ABC Curve of medicines in a Basic Health Unit of the city of Belém-PA, showing its relevance for efficient stock control. Methods: It's a descriptive cross-sectional study that used information from drug management utilized at $\mathrm{BHU}$, through data collected from monthly drug requests from September 2017 to August 2018. Data were analyzed using Microsoft Office software Excel® 2013. Results: of the 268 standardized drugs, 105 were absent. Of the 112 in the primary care inventory, class A accounted for $18.42 \%$ of these drugs representing $69.76 \%$ of the costs, class B $21.05 \%$ of the items and represented $19.25 \%$ of expenses, C $60.53 \%$ of items with $10.99 \%$ of costs. In the urgency and emergency of the 51 drugs, classes $A$ and $B$ corresponded to $14.29 \%$ of drugs and class $C$ to $71.43 \%$. The financial demand was: $68.93 \%(A)$, $20.26 \%$ (B) and $C$ of $10.81 \%$. Conclusion: It's important to start investing drug management, to rationalize resources, pharmacological therapy, and to avoid breaking the stock.
\end{abstract}

Keywords: Hospital materials management, Health management, Pharmaceutical care.

\footnotetext{
${ }^{1}$ Universidade da Amazônia (UNAMA), Belém-PA. *E-mail: jessicanadc@gmail.com

2 Faculdade Estácio de Belém, Belém-PA.

${ }^{3}$ Laboratório Sabin, Belém - PA.

${ }^{4}$ Secretaria Municipal de Saúde de Ananindeua, Ananindeua - PA.

${ }^{5}$ Hospital Ophir Loyola, Belém - PA.

6 Universidade Federal do Pará, Belém - PA.
} 


\section{RESUMEN}

Objetivo: Elaborar la Curva $A B C$ de medicamentos en una Unidad Básica de Salud de la ciudad de BelémPA, mostrando su relevancia para el control eficiente de las existencias. Métodos: Este es un estudio descriptivo de corte transversal se utilizó información de medicamentos utilizada en la UBS, a través de datos recopilados de solicitudes mensuales de los de septiembre de 2017 a agosto de 2018. Los datos se analizaron con el software de Microsoft Office Excel® 2013. Resultados: De los 268 medicamentos estandarizados, 105 estaban ausentes. De los 112 en el inventario de atención primaria, la clase A representaba el $18.42 \%$ de estos medicamentos que representan el $69.76 \%$ de los costos, la clase B el $21.05 \%$ de los artículos y representó el $19.25 \%$ de los gastos, C $60.53 \%$ de los artículos con $10.99 \%$ de los costos. En la urgencia y emergencia de las 51 drogas, las clases A y B correspondieron al $14.29 \%$ de las drogas y la clase $\mathrm{C}$ al $71.43 \%$. La demanda financiera fue: $68.93 \%$ (A), $20.26 \%$ (B) y C de $10.81 \%$. Conclusión: Es importante invertir en el manejo de medicamentos, racionalizar los recursos, la terapia farmacológica y evitar romper el stock.

Palabras clave: Gestión de materiales hospitalarios, Gestión sanitaria, Atención farmacéutica.

\section{INTRODUÇÃO}

De acordo com Sistema Único de Saúde (SUS) criado pela Constituição Federal de 1988, através das resoluções geradas na $8^{\text {a }}$ Conferência Nacional de Saúde realizada em 1986, e regulamentado pela Lei orgânica ํㅡ 8.080 de 19 de setembro de 1990, todo cidadão tem seu direito garantido à saúde, cabendo ao Estado executá-lo por meio do (SUS), bem como a assistência farmacêutica e terapêutica integralmente. Porém, assegurar o acesso ao medicamento no país tornou-se uma das questões de prioridades nacionais de saúde (GARCIA MM, et al., 2017).

A crescente demanda de financiamento para aquisição de medicamentos no setor público vem tornandose um impasse, pois trava uma disputa com outras prioridades da área. Além de tudo, esses gastos não têm refletido substancialmente em melhoria ao acesso à saúde da população, provocando ameaça à sustentabilidade dos sistemas públicos de muitos países (FALEIROS DR, et al., 2017).

Estes custos podem ser contidos sem diminuição da qualidade do serviço que está destinado a fazer, através da elaboração de sistemas de gerenciamento de gastos (LOURENÇO KG e CASTILHO V, 2006). Visto que, o aumento das despesas farmacêuticas pode ser em função do alto valor unitário de alguns medicamentos ou pelo alto volume de itens necessários para atender as necessidades da população (FALEIROS DR, et al., 2017).

Nesse contexto, a estruturação da Assistência Farmacêutica (AF) no SUS nos últimos anos, vem sendo encarada como uma estratégia essencial capaz de melhorar o acesso da população ao medicamento e para consolidação do próprio sistema de saúde (LEITE SN, et al., 2016).

Segundo Vieira FS (2010), a AF foi definida pela Política Nacional de Medicamentos (PNM) como um conjunto de atividades ligadas ao medicamento, com objetivo de apoiar as ações de saúde necessárias à comunidade. Ainda segundo o autor, apesar dos avanços com a incorporação da AF no SUS, para que as políticas criadas pelo Estado sejam executadas, faz-se necessário a disponibilização de recursos suficientes para a efetivação das atividades e de uma gestão capacitada.

Conforme a PNM, a reorientação da AF está fundamentada na descentralização da gestão, na promoção do uso racional dos medicamentos, na otimização e eficácia do sistema de distribuição no setor público e no desenvolvimento de iniciativas que possibilitem a redução nos preços dos produtos (BRASIL, 2001).

A estruturação e organização da $A F$ tem dado preferência às ações no nível da macrogestão municipal, desenvolvendo atividades técnicas relacionadas ao ciclo logístico do medicamento: seleção, programação, aquisição, armazenamento, distribuição e dispensação.

O bom funcionamento deste ciclo tem uma grande importância, pois através dele os gestores em saúde aperfeiçoam o uso dos recursos (financeiros, humanos, etc.) e qualificam o medicamento que dispõem (PEREIRA NC, et al., 2015). 
Segundo Vecina Neto G e Filho WR (1998), a gestão de materiais tem como propósito destinar os recursos necessários ao desempenho produtivo com eficiência, quantidade e tempo certo, sem gastos desnecessários. Ainda conforme o autor, na área da saúde todo item consumido é importante, porém, fazendo uma análise dos gastos relacionados com a aquisição de cada item de estoque possuem diferenças entre si. Com a demanda de medicamentos sendo aleatória e a variedade desses insumos significativa, é importante que o gestor organize os materiais em grupos semelhantes, permitindo a individualização das estratégias relacionadas a esses produtos.

A administração de medicamentos para o setor público de saúde possui uma deficiência para os governos, tendo e vista que a falta de um mecanismo eficiente de controle impossibilita o levantamento dos materias necessários para o reabastecimento, calculando-se orçamento na maioria das vezes inferior do necessário para suprir a demanda da instituição.

Outro fator importante é a perda de materias por expiração do prazo de validade, a falta do controle dos mesmos e a inexistência de rastreabilidade da movimentação desses itens pela rede, submete o sistema público de saúde à posição de fragilidade, sujeito de críticas e ataques por parte dos usuários (TEIXEIRA PB, 2010).

Nesse cenário, a classificação pelo método $A B C$ é uma ferramenta que classifica estatisticamente os materiais, levando em consideração a sua importância, de acordo com a quantidade utilizada e o seu valor unitário. Esta permite analisar com clareza as ações desenvolvidas por uma organização, podendo melhorar a eficiência de custos, pois fornece informações necessárias sobre os gastos, resultando no seu melhor gerenciamento e redução das despesas (POPESKO B e NOVÁK P, 2011).

Em geral, são colocados, no máximo, $20 \%$ dos itens na classe A (estes representam uma demanda de $70 \%$ dos recursos num dado período), $30 \%$ na classe $B$ (com uma demanda de recursos de $20 \%$ num dado período) e $50 \%$ na classe C (com uma demanda de recursos de apenas $10 \%$ num dado período) (GOMES MJVM e REIS AMM, 2003).

Dessa forma, este estudo pretende demonstrar a relevância da elaboração da curva $A B C$ de medicamentos em uma Unidade Básica de Saúde (UBS) na capital do Estado do Pará, para o controle de estoque eficiente.

\section{MÉTODOS}

Trata-se de um estudo descritivo, com delineamento retrospectivo, transversal com abordagem quantitativa, realizada na Central de Abastecimento Farmacêutico em Belém do Pará, Brasil.

A UBS na qual foi realizada o estudo, foi escolhida por atender uma ampla variedade de consultas em várias especialidades, bem como urgência e emergência, pediatria, ginecologia, clínica geral, endocrinologia, odontologia, além dos atendimentos prestados pela equipe de enfermagem na baixa, média e alta complexidade, entre outros. Sendo assim, a UBS dispõe de grande variedade de medicamentos.

Para o desenvolvimento deste trabalho, foram utilizadas informações referentes ao gerenciamento de medicamento utilizados na UBS, dados foram coletados no período de setembro, outubro e dezembro de 2018, referente a setembro de 2017 a agosto de 2018, totalizando um período de um ano.

Em relação à técnica de coleta de dados, às informações foram obtidas através de cópias das requisições mensais de medicamentos gerais, que continham dados referentes aos medicamentos, tais como: nome genérico dos fármacos, valor unitário, quantidade solicitada e quantitativo que a unidade foi atendida. Após coletados, os dados foram tabulados no Microsoft Excel $\Theta 2013$, no qual foram realizados os agrupamentos por classe terapêutica para posterior elaboração da Curva ABC, de acordo com o método proposto (GOMES MJVM e REIS AMM, 2003).

Foram levantados os seguintes dados: relação dos itens, custo unitário e consumo anual de cada item, sendo estes posteriormente tabulados no Microsoft Excel® 2013. Em seguida, os itens foram relacionados, conforme seu custo unitário e o consumo anual, através do produto destes valores foi obtido o custo anual. 
Os medicamentos foram listados conforme o valor decrescente da despesa anual. Posteriormente foi realizado o cálculo para obtenção dos custos anuais acumulados, através da soma do valor de cada item, anotando os valores até a obtenção do valor total. A partir destes se estabelecem as porcentagens, logo os medicamentos foram agrupados em classes ( $A, B$ e C).

Por ser um estudo que utilizou como fonte de dados requisições de medicamentos gerados pelo Sistema Nacional de Gestão da Assistência Farmacêutica - HÓRUS, conforme os autores Costa KS e Nascimento Jr JMD (2012), é um sistema de informação web disponibilizado para aos municípios, estados e Distrito Federal - o qual contém informações gerenciais que contribuem com o gerenciamento e desenvolvimento da atuação da AF na Atenção Básica, e estas sendo de domínio público, e não havendo dados primários ou secundários de usuários, não houve a necessidade de submissão da mesma a apreciação do Comitê de Ética em Pesquisa (CEP-CONEP).

\section{RESULTADOS}

Sabe-se que, é de responsabilidade do Ministério da Saúde a publicação de uma lista de medicamentos fundamentais para tratar as doenças mais comuns na população brasileira, chamada de Relação Nacional de Medicamentos essências (RENAME). A partir desta, os municípios elaboram suas próprias listas, denominadas de Relação Municipal de Medicamentos (REMUME), baseadas em critérios epidemiológicos locais, sazonalidades e outros (BRASIL, 2001).

A lista padrão de medicamentos da Unidade Municipal em estudo é composta por 268 medicamentos, porém foi observado a ausência de 105 medicamentos destes. Logo, no total foram coletados dados de 163 medicamentos, que foram listados e Tabelados no Microsoft Excel $\AA^{2} 2013$, de acordo com o seu nome genérico, forma farmacêutica, quantidade consumida no período estudado e valor unitário de cada medicamento.

Posteriormente, para facilitar a visualização, dividiu-se essa lista em duas: medicamentos da atenção básica de saúde, com 112 fármacos agrupados em 38 classes terapêuticas, e medicamentos utilizados na urgência e emergência da UBS, com 51 medicamentos agrupados em 21 classes. Após serem agrupados em classes terapêuticas foram submetidos ao cálculo conforme o método já mencionado (GOMES MJVM e REIS AMM, 2003) para classificação A, B e C (Tabelas 1 e 2).

Tabela 1 - Divisão de Classe dos medicamentos da atenção básica de saúde.

\begin{tabular}{ccccc}
\hline Classe & A & B & C & TOTAL \\
\hline Quantidade de itens & 7 & 8 & 23 & 38 \\
\% Itens & 18,42 & 21,05 & 60,53 & 100,00 \\
Valor classe & $\mathrm{R} \$ 77.405,22$ & $\mathrm{R} \$ 21.358,96$ & $\mathrm{R} \$ 12.195,81$ & $\mathrm{R} \$ 110.959,99$ \\
$\%$ Valor & 69,76 & 19,25 & 10,99 & 100,00 \\
\hline
\end{tabular}

Fonte: Costa JNA et al., 2019.

Tabela 2 - Divisão de Classe dos medicamentos utilizados na urgência e emergência.

\begin{tabular}{ccccc}
\hline Classe & A & B & C & TOTAL \\
\hline Quantidade de itens & 3 & 3 & 15 & 21 \\
$\%$ Itens & 15,79 & 15,79 & 68,42 & 100,00 \\
Valor classe & $\mathrm{R} \$ 73.800,10$ & $\mathrm{R} \$ 22.256,87$ & $\mathrm{R} \$ 11.010,60$ & $\mathrm{R} \$ 107.067,57$ \\
$\%$ Valor & 68,93 & 20,79 & 10.28 & 100,00 \\
\hline
\end{tabular}

Fonte: Costa JNA, et al., 2019. 
Analisando os medicamentos da atenção básica de saúde, foi possível notar que sete classes de fármacos são classificadas como $A$ na curva $A B C$, sendo eles: antimicrobianos, antidiabéticos, antifúngicos, antihipertensivos, anticoncepcionais, os anti-inflamatórios não esteroides e os hipolipemiantes. As classes de medicamentos agrupados como B foram: corticoides, expectorantes, antiácidos, poli-vitamínicos, antihelmínticos, protetores gástricos e anti-histamínicos e os da classe $C$ foram: antianêmicos, antivirais, analgésicos, broncodilatadores, diuréticos, repositores hormonais, bisfosfonatos, antigases, reguladores intestinais, vasodilatadores, antiparasitários, antieméticos, digitálicos, laxantes, pomadas ginecológicas e outros, como demostrado abaixo (Gráfico 1).

Gráfico 1 - Classificação $A B C$ dos medicamentos usados na atenção básica de saúde.

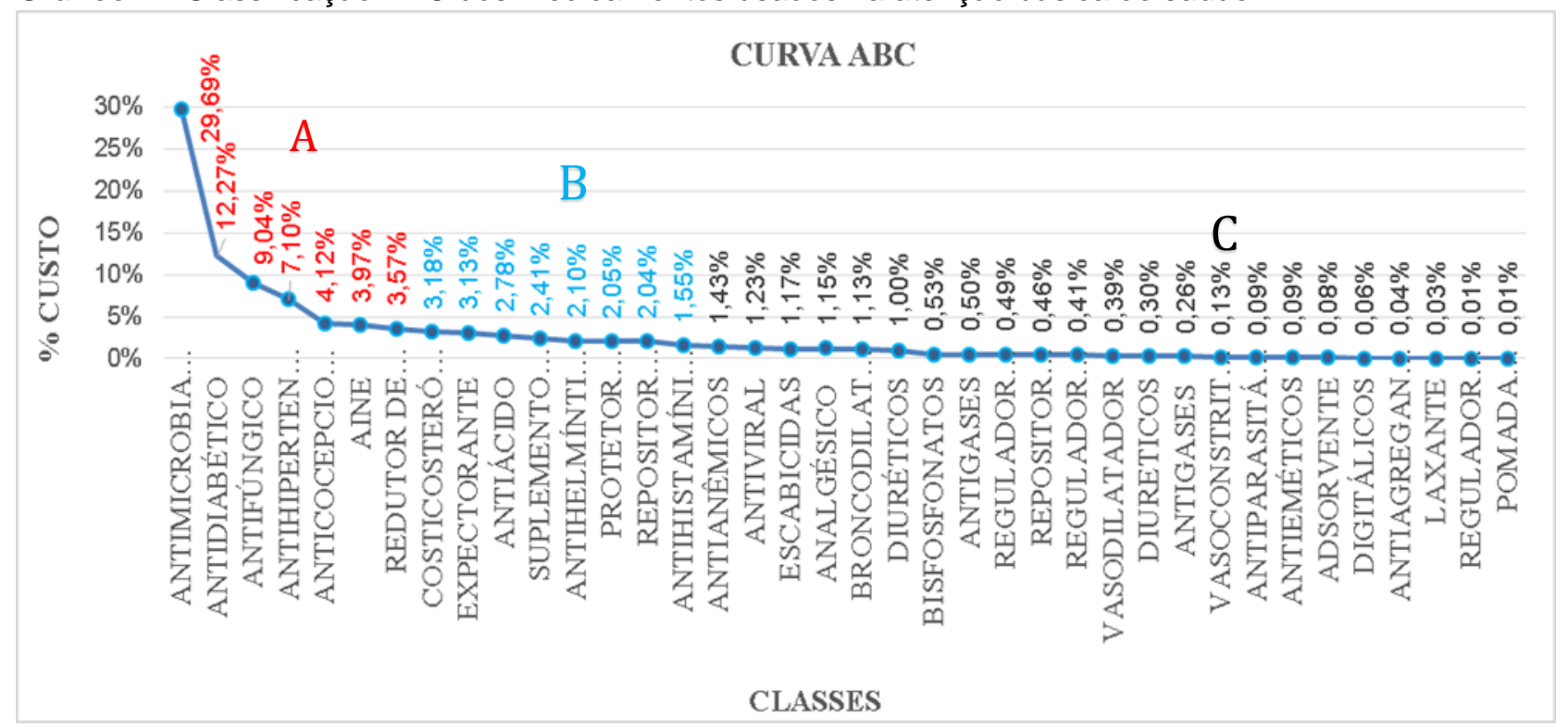

Fonte: Costa JNA et al., 2019.

Quanto aos medicamentos da urgência e emergência, foram agrupados em A: Anti-inflamatórios não esteroidais (AINE's), antimicrobianos e corticoides, da classe B foram: repositores eletrolíticos, antiespasmódicos e analgésicos, além de antitérmicos e os da classe C: anti-histamínicos, polivitamínicos, relaxantes musculares, antieméticos, diuréticos, hipertensores, anestésicos locais, anti-hemorrágicos, antiarrítmicos, digitálicos, expectorantes e parassimpaticolíticos, como demostrado no Gráfico 2.

Gráfico 2 - Classificação ABC de medicamentos utilizados na urgência e emergência.

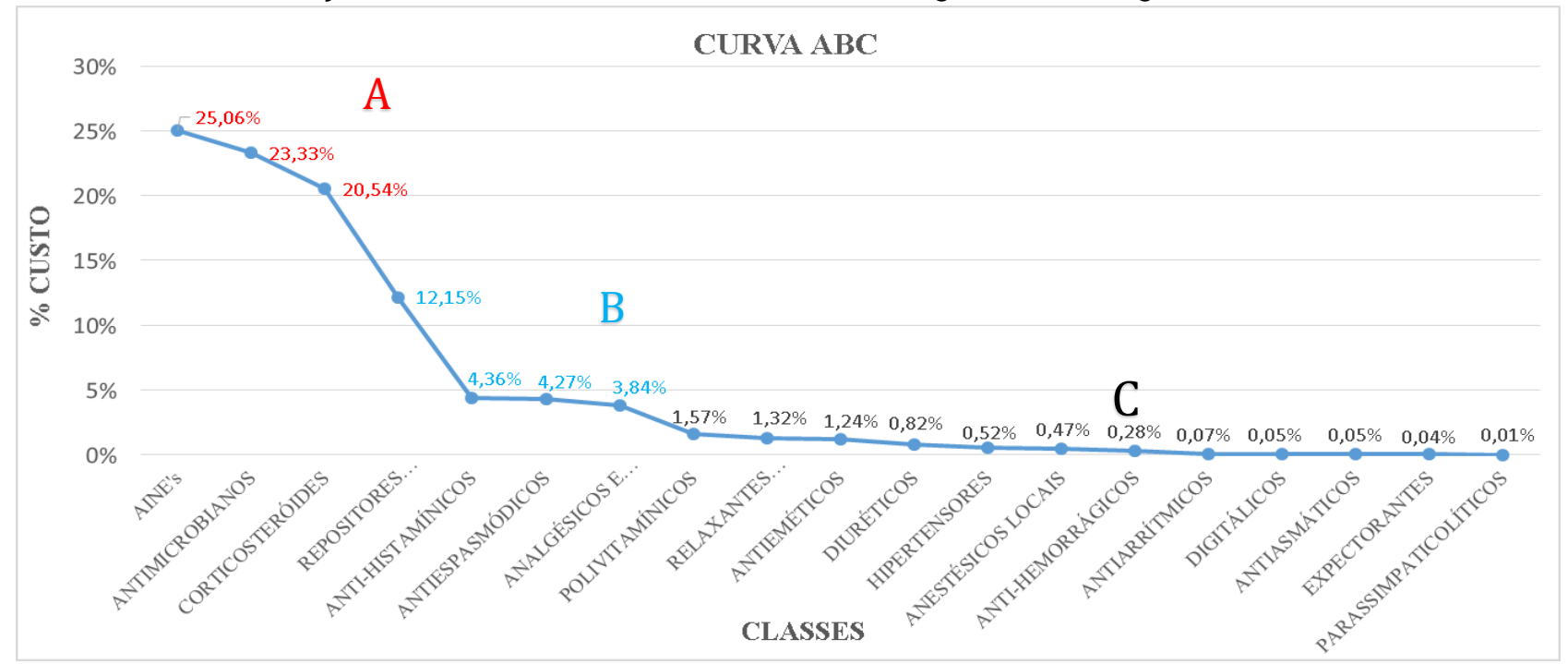

Fonte: Costa JNA et al., 2019. 


\section{DISCUSSÃO}

Considerando que é necessário realizar a padronização dos medicamentos e/ou materiais utilizados em unidades básicas de saúde, com o intuito de otimizar os estoques (CAVALLINI ME e BISSON MP, 2002), estudos que incentivem e demonstrem o controle de estoque são relevantes. Sabe-se que esta padronização pode apresentar diferenças de acordo com as peculiaridades de cada serviço.

Segundo Mendes EV (2002), a atenção primaria a saúde tem três funções: resolução, coordenação e responsabilização, enquanto o setor de urgência e emergência tem um perfil de atendimento de situações que necessitam de atendimento imediato (MEDEIROS NJS, 2016).

Neste estudo, realizou-se a elaboração de Curva $A B C$ de dois setores dentro da UBS: o de medicamentos básicos e de urgência e emergência, onde detectou-se perfis diferentes de medicamentos e gestão de estoque. Do total de medicamentos padronizados pela unidade de saúde, todos requerem atenção, conforme os autores Castilho KG e Lourenço V (2006), porém não é aconselhável dar o mesmo grau de importância a todos os itens do estoque, pois observa-se diferença nas peculiaridades de cada um, como valor unitário, demanda de consumo, prazo de entrega entre outros.

Assim sendo, o total de medicamentos padronizados pela unidade de saúde foram de 268, porém observou-se a ausência de 105 medicamentos. Logo, estes ficaram desabastecidos em todo o período de estudo. Tal fato demonstra falha no ciclo logístico do medicamento, muito provavelmente em função da verba disponível, que geralmente é escassa e devido a falhas no planejamento da logística farmacêutica. Logo, foram coletados dados de apenas 163 fármacos, divididos entre atenção básica e urgência e emergência.

Nesse sentido, Vecina Neto e Malik AM (2012), relatam que para evitar o desabastecimento e compras antes do período certo, é necessário atentar-se ao ponto de pedido para realizar compras novas enquanto a quantidade de itens armazenados no estoque for ideal para suprir as demandas da empresa do período entre a solicitação e a chegada do pedido. Além disso, é necessário que haja um estoque reserva, que é uma quantidade de material suficiente para suprir possíveis necessidades como atrasos dos fornecedores na entrega.

Dos 112 itens do estoque da atenção básica, a classe A correspondeu a 18,42\% destes medicamentos e representou uma demanda de alocação de recurso de $69,76 \%$, a classe B correspondeu a $21,05 \%$ dos itens e representou uma demanda financeira de $19,25 \%$, já a classe C correspondeu a $60,53 \%$ do total dos itens e demandou 10,99\% de custos com estes. Já na urgência e emergência, dos 51 medicamentos, as classes $A$ e B corresponderam a $14,29 \%$ dos fármacos do estoque e a classe C a 71,43\%.

Enquanto a demanda financeira de cada classe foi de: $68,93 \%$ (A), $20,26 \%$ (B) e a C de $10,81 \%$. Estes dados corroboram com os obtidos no estudo de Bauer AL (2015) realizado em um hospital do SUS da cidade de Novo Hamburgo - RS, que apesar de conter maior número de medicamentos padronizados, 372 itens, encontrou-se resultados semelhantes: a classe A correspondeu 6,2\% dos itens, representando $75,9 \%$ do total de custos, a classe B $12,9 \%$ dos itens que representavam $17,2 \%$ dos gastos, e $80,9 \%$ dos itens representavam a classe $\mathrm{C}$, responsáveis por $6,9 \%$ dos gastos.

Analisando os dados de consumo dos medicamentos apresentados nas Tabelas 1 e 2, verificou-se que o valor total gasto na Unidade Municipal de Saúde com estes insumos no período analisado foi de $R \$$ 218.027,56. Levando em consideração que a Classe A demanda maior porcentagem do gasto total e, por isso, maior importância administrativa, separou-se esta classe para uma análise mais profunda.

Nota-se que a classe A referente aos dois perfis demonstrados no Gráficos 1 e 2, foi representada pelas classes terapêuticas antimicrobianos, antidiabéticos, antifúngicos, anti-hipertensivos, anticoncepcionais, antiinflamatórios não esteroides e hipolipemiantes. Destas, os antimicrobianos foram os mais importantes, tanto na curva da atenção básica, ocupando o primeiro lugar, (com custo anual de $R \$ 32$. 943, 92), quanto na urgência e emergência, a qual ocupou o segundo lugar da Curva, demandando um gasto anual de $R \$ 26$. 832, 00. Esse dado explica-se pelo fato de os medicamentos da referida classe possuírem um alto valor unitário aliado a uma alta demanda de consumo, corroborando com o estudo dos autores De Sousa Lima HK, et al. (2018), que afirma que os antimicrobianos representam uma das classes terapêuticas mais distribuída 
e utilizada nos serviços da saúde e em relação aos custos, foi constatado que os gastos com antibióticos para a atenção básica são altos. Logo, necessitam de muita atenção dos gestores, principalmente, com relação à sua quantidade em estoque e validade.

De acordo com o Gráfico 1, referente ao perfil da atenção básica, os antidiabéticos ocuparam a segunda posição da classe A comprometendo $12,27 \%$ do orçamento. Esse dado justifica-se pelo fato de que tanto a Insulina Regular quanto a Insulina NPH, que fazem parte dessa classe terapêutica, possuem um alto valor unitário associado a um alto consumo. Corroborando com o estudo de Mattos GOG, et al. (2013), realizado em uma farmácia municipal de Florianópolis, que apesar de seus itens não estarem organizados por classes terapêuticas, esses dois medicamentos também foram classificados como Classe A.

Ainda conforme o Gráfico 1, os antifúngicos também classificados como $\mathrm{A}$, ocuparam a terceira posição comprometendo $9,04 \%$ dos recursos. Esse dado mostra que apesar de a maioria dos medicamentos desta classe possuírem valor unitário relativamente baixo como, por exemplo, o Fluconazol cápsula de 150mg com valor unitário de $R \$ 0,19$ teve 1700 unidades dispensadas no período analisado. Esse resultado mostra que os itens de baixo valor unitário também podem possuir alto impacto financeiro e pertencer a Classe $A$, isso ocorre em virtude da sua alta demanda de consumo, e por isso merecem uma atenção especial dos gestores.

Com relação ao Gráfico 2, referente ao perfil da Urgência e Emergência, a classe terapêutica AINE ocupou o primeiro lugar na Curva A, sendo responsável por $25,06 \%$ dos gastos. Esse dado já era esperado pelo fato da alta demanda de consumo, o que é consistente com estudos que mostram que essa classe terapêutica representa uma das mais utilizada no mundo, como mostra os autores Bandeira VAC, et al. (2013) em um estudo realizado em uma unidade básica de saúde no município de ljuí/RS, no qual verifica-se que a classe terapêutica foi a mais prescrita.

Conforme Gomes MJVM e Reis AMM (2003), os materiais da classe A possuem maior impacto financeiro sendo responsáveis pela maior parte do investimento, devido a isso deve-se manter um estoque de segurança reduzido, porém deve-se assegurar uma quantidade suficiente para evitar o desabastecimento. Ainda segundo os autores, é prudente manter alto índice de rotatividade, deixando, assim, o capital de giro maior e evitando bloqueio de recursos. Em relação aos itens da Classe B, mesmo possuindo menor impacto financeiro com importância financeira intermediária, é recomendado deixar um estoque reduzido e, também, maior índice de rotatividade. Enquanto que, os itens classificados como curva $\mathrm{C}$, existe a possibilidade de aumento dos períodos de aquisição desses materiais, elevando os níveis dos materiais armazenados, o que favorece a negociação com os fornecedores e diminui os gastos de aquisição desses itens.

Através da análise da Curva ABC, percebe-se o grau de importância de cada item para o estoque, e assim permite ao farmacêutico ter um maior controle dos materiais disponíveis na farmácia e CAF. Esta caracterização permite ainda saber a demanda destes insumos e assim racionalizar o uso dos recursos, de modo que se adquiram quantidades suficientes para atender a demanda dos serviços, evitando perdas, compras emergenciais e principalmente a ruptura do estoque.

Quanto a disponibilidade de estudos na literatura para comparação do presente trabalho, ressalta-se que há um número escasso, sendo necessário a realização de mais estudos de gestão de custos voltados à área da saúde, principalmente no que tange a atenção básica. Observa-se ainda que a maioria dos autores têm abordagem dos processos a nível hospitalar e industrial.

\section{CONCLUSÃO}

Este estudo evidenciou a importância do gerenciamento adequado de medicamentos para a racionalização dos recursos e melhoria da qualidade dos serviços ofertados pela UBS. A Curva ABC é uma excelente ferramenta de gestão de estoque, que possibilitou a visualização das particularidades de cada medicamento em relação ao seu impacto financeiro. Assim, possibilita um melhor planejamento em saúde para os gestores do SUS. Ressalta-se a importância de investir na gestão dos recursos e capacitação dos profissionais da saúde para a minimização dos problemas detectados neste estudo. Destaca-se ainda a escassez de estudos acerca desta temática, especialmente à nível de atenção básica. Portanto, esta pesquisa contribuirá para a geração de informações acerca da gestão de custos aplicados na área da saúde no nível de atenção básica. 


\section{REFERÊNCIAS}

1. BANDEIRA VAC, et al. Uso de anti-inflamatórios não esteroides por idosos atendidos em uma Unidade de Estratégia de Saúde da Família do município de ljuí (RS). Revista Brasileira de Ciências do Envelhecimento Humano, 2013; 10 (2): 181-192.

2. BAUER AL. Gestão da assistência farmacêutica: aplicação da curva ABC para gestão de medicamentos em uma farmácia hospitalar do Sistema Único de Saúde. Dissertação (Especialização de Gestão em Saúde) - Programa Nacional de Formação em Administração Pública. Universidade Federal do Rio Grande do Sul, Novo Hamburgo, 2015; 20 p.

3. BRASIL, Ministério da Saúde. Política Nacional de Medicamentos. Brasília: DF, 2001. Série C, n. 25.

4. CAVALLINI ME e BISSON MP. Farmácia Hospitalar - um enfoque em sistemas de saúde. Barueri: Manole, 2002; $304 p$.

5. COSTA KS, NASCIMENTO Jr JMD. HÓRUS: inovação tecnológica na assistência farmacêutica no Sistema Único de Saúde. Revista de Saúde Pública, 2012; 46 (1): 91-99.

6. DE SOUSA LIMA HK, et al. Distribuição e custo de antimicrobianos na Atenção Primária. Acta Paulista de Enfermagem, 2018; 31(1): 95-101.

7. FALEIROS DR, et al. Financiamento da assistência farmacêutica na gestão municipal do Sistema Único. Revista de Saúde Pública, 2017; 51 (2): 14.

8. GARCIA MM, et al. Avaliação econômica dos programas Rede Farmácia de Minas do SUS versus Farmácia Popular do Brasil. Ciência \& saúde coletiva, 2017; 22 (1): 221-233.

9. GOMES MJVM, REIS AMM. Ciências Farmacêuticas: uma abordagem em farmácia hospitalar. São Paulo: Atheneu, 2003; 370-372 p.

10. LEITE SN, et al. Gestão da assistência farmacêutica. Florianópolis-SC: UFSC, 2016; 41p.

11. LOURENÇO KG, CASTILHO V. Classificação ABC dos materiais: uma ferramenta gerencial de custos em enfermagem. Revista Brasileira de Enfermagem, 2006; 59 (1): 52-55.

12. MATTOS GOG, et al. Gestão e classificação ABC dos medicamentos padronizados pela prefeitura municipal de Florianópolis. Coleção Gestão da Saúde Pública, 2013; 5 (1): 56-78.

13. MEDEIROS NJS. Acolhimento às urgências e emergências na atenção básica: intervenções e propostas da unidade Santo Antônio - Coronel Ezequiel, RN. Trabalho de Conclusão de Curso (Pós-Graduação em Atenção Básica em Saúde), São Luís, 2016; 6-18.

14. MENDES EV. Os sistemas de serviços de saúde: o que os gestores deveriam saber sobre essas organizações complexas. Fortaleza: Escola de Saúde Pública do Ceará, 2002.

15. PEREIRA NC, et al. Serviços farmacêuticos na atenção primária no município do Rio de Janeiro: um estudo de avaliabilidade. Saúde em Debate, 2015; 39 (1): 451-468.

16. POPESKO B, NOVÁK P. Application of ABC method in hospital management. Proceedings of the 6th IASME/WSEAS International Conference on Ecponomy and Management transformation, 2011; 11 (1): 17-19.

17. TEIXEIRA PB. Diagnóstico das condições de armazenamento de medicamentos em um almoxarifado municipal. Trabalho de Conclusão de Curso (graduação em Farmácia). Universidade do Extremo Sul Catarinense, UNESC Criciúma, 2010; 35 p.

18. VECINA NETO G, FILHO WR. Para gestores municipais de serviços de saúde. Gestão de Recursos Materiais e de Medicamentos Gonzalo Vecina. Instituto para o desenvolvimento da saúde - IDS Núcleo de Assistência MédicoHospitalar - NAMH/FSP - USP Banco Itaú São Paulo 1998.

19. VECINA NETO G, MALIK AM. O futuro dos serviços de saúde no Brasil. Rio de Janeiro: Guanabara Koogan, 2012; 351-7p.

20. VIEIRA FS. Assistência farmacêutica no sistema público de saúde no Brasil. Revista Panamericana de Saúde Pública, 2010; 27 (1): 149-156. 